\title{
Ultrasound-assisted extraction kinetics, fatty acid profile, total phenolic content and antioxidant activity of green solvents extracted passion fruit oil
}

\begin{abstract}
The main objective of this current work was to evaluate the feasibility of green solvent's application on passion fruit oil extraction. In this study, the effects of green solvent on oil yield, fatty acid profile, oil physicochemical properties, total phenolic content and antioxidant activity associated with extracted oil were compared to those of conventional solvent, hexane application. Therefore, this study may provide supplementary information on previous studies that focused on oil recovery. Oilseeds' proximate composition and the influence of oilseedsto-solvent $(\mathrm{w} / \mathrm{v})$ ratio on oil yield were investigated. The oilseeds contained high fibre, fat and protein. Oilseeds-to-solvent (w/v) ratio (1:8) provided the highest oil yield. Overall, acetone was suggested as a suitable hexane replacer due to its higher oil recovery, similar fatty acid profile and oil physicochemical properties with higher antioxidant activity. Ethanol-extracted oil contained higher amounts of omega-9 MUFA, which may indicate the influence of extraction solvent on final fatty acid composition and thus final oil application.
\end{abstract}

Keyword: Fatty acids; Lipids; Oilseeds 